\title{
High distance knots
}

\author{
YAIR N MINSKY \\ YOAV MORIAH \\ SAUL SCHLEIMER
}

\begin{abstract}
We construct knots in $S^{3}$ with Heegaard splittings of arbitrarily high distance, in any genus. As an application, for any positive integers $t$ and $b$ we find a tunnel number $t$ knot in the three-sphere which has no $(t, b)$-decomposition.
\end{abstract}

57M25, 57M27

\section{Introduction}

In this paper we address the problem of generating knots in $S^{3}$ with high distance, in the sense of Hempel. Without any restriction on the ambient manifold, knots with high distance are easy to construct: Hempel [9], adapting an idea of Kobayashi [13], constructs Heegaard splittings $(V, W)$ of arbitrarily high distance. Given such a splitting remove an unknotted solid torus from, say, $V$ to obtain a compression body $V_{0}$. The result is a knot space in some manifold $M$ and clearly the distance of the Heegaard splitting $\left(V_{0}, W\right)$ is at least the distance of $(V, W)$. See Section 2 for definitions.

The problem becomes more challenging if the ambient manifold $M$ is specified beforehand. The argument above fails if $M$ does not admit any high distance splitting. The case of $S^{3}$ is of particular interest, and here we know (see Waldhausen [24]) that every Heegaard splitting is isotopic to a standard one. The disk complexes for a standard splitting have distance zero, and indeed an infinite-diameter intersection. This makes finding an appropriate compression body more difficult. Nevertheless, we prove:

Theorem 3.1 For any pair of integers $g>1$ and $n>0$ there is a knot $K \subset S^{3}$ and a genus $g$ splitting of its exterior $E(K)$ having distance greater than $n$.

We can consider another measure of complexity of a knot: $K \subset M$ has a $(g, b)-$ decomposition if $K$ may be isotoped to have exactly $b$ bridges with respect to a genus $g$ Heegaard splitting of $M$. When $b=0$ we further insist that $K$ be a core of one of the handlebodies (see Remark 2.4). The classical notion of "bridge position" corresponds to a $(0, b)$-decomposition. 
Work of Scharlemann-Tomova [20] and Tomova [23; 22] links these two notions of complexity. It implies in particular that the existence of a $(g, b)$-decomposition gives upper bounds on the distance of Heegaard splittings of genus greater than $g$ (see Section 4 for details). With this we obtain the following consequence of Theorem 3.1:

Theorem 4.2 For any positive integers $t$ and $b$ there is a knot $K \subset S^{3}$ with tunnel number $t$ so that $K$ has no $(t, b)$-decomposition.

This theorem answers a question of Kobayashi-Rieck [15, Question 1.9]. Note that any knot $K$ with tunnel number $t$ (see Section 2) has by definition a $(t+1,0)-$ decomposition. The corresponding splitting surface of $E(K)$ has minimal genus. In Kobayashi-Rieck [14], these results are used to disprove Morimoto's conjecture on subadditivity of Heegaard genus.

Previous constructions have yielded tunnel number $t$ knots without $(t, 1)$-decompositions (Moriah and Rubinstein [17], Morimoto, Sakuma, and Yokota [18], and Eudave-Muñoz [6]). The knots described in [17] have $(t, 2)$-decompositions. The knots in [18] are all tunnel number one knots and have $(1,2)$-decompositions. The knots in [6] are all tunnel number one and some are known to be $(1,2)$ while the rest have unknown optimal $(1, b)$-decomposition.

More recently Eudave-Muñoz [5] has exhibited tunnel number one knots in $S^{3}$ which are not $(1,2)$. His examples are either $(1,3)$ or $(1,4)$ knots but exactly which is not yet known.

Johnson and Thompson have shown, in [11] (see also Johnson [10]), that for arbitrarily large $n$ there are tunnel number one knots which have no $(1, n)$-decomposition. Their proof also uses the Scharlemann-Tomova results to relate Heegaard distance to bridge position. Constructing high distance tunnel number one knots is simplified by the special properties of disk complexes in genus 2 compression bodies - see the discussion in Section 3.

\section{Further questions}

One is tempted to speculate that Theorem 3.1 holds for any 3-manifold. We note however that our construction fails for the standard Heegaard splitting of a connected sum of $S^{2} \times S^{1}$,s, because the disk complexes on both sides of that splitting are identical. A more plausible conjecture is that, after stabilizing once or possibly twice, any Heegaard splitting will admit a construction like the one we have used. 


\section{Acknowledgements}

This work arose out of conversations between the authors at the Workshop on Heegaard Splittings of 3-Manifolds, held at the Technion in Haifa (Israel), July of 2005. Further work was done at Rutgers and Yale University during February of 2006. We wish to thank the Technion, Rutgers, and Yale University for their hospitality. This work was partially supported by NSF grants DMS-0504019 and DMS-0508971, and Grant No. 2002039 from the US-Israel Binational Science Foundation (BSF). It is in the public domain.

\section{Preliminaries}

A compression body is a 3-manifold $V$ obtained from a surface $S$ cross an interval $[0,1]$ by attaching a finite number of 2-handles and 3-handles to $S \times\{0\}$. The component $S \times\{1\}$ of the boundary will be denoted by $\partial_{+} V$, and $\partial V \succ_{+} V$ will be denoted by $\partial_{-} V$. The trivial cases where $V$ is a handlebody or $V=S \times[0,1]$ are allowed.

A Heegaard splitting for a 3-manifold $M$ is a decomposition $M=V \cup_{S} W$ where $V, W$ are compression bodies so that $S=\partial_{+} V=\partial_{+} W=V \cap W$. The surface $S$ will be called the Heegaard surface of the Heegaard splitting.

\subsection{Complexes}

Let $C_{S}$ be the 1-skeleton of Harvey's complex of curves (see [7]). That is, given a closed connected orientable surface $S$ of genus at least two, let $\mathcal{C}_{S}$ be the graph whose vertices are isotopy classes of essential simple closed curves and whose edges connect distinct vertices with disjoint representatives. As usual, we only distinguish curves from their isotopy classes when necessary.

We remark that $\mathcal{C}_{S}$ is connected. Place a metric $d(\cdot, \cdot)$ on $\mathcal{C}_{S}$ by setting the length of every edge to be one. For subsets $X, Y \subset \mathcal{C}_{S}$ we define $d(X, Y)=\min \{d(x, y) \mid x \in$ $X, y \in Y\}$.

An essential curve $\alpha \subset S=\partial_{+} V$ is a meridian of the compression body $V$ if $\alpha$ bounds an essential disk in $V$. Given a compression body $V$ so that $\partial_{+} V=S$, let $\mathcal{D}_{V}$ be the sub-complex of $\mathcal{C}_{S}$ spanned by meridians of $V$. This is the one-skeleton of McCullough's disk complex (see [16]).

Hempel [9] defined the distance of a Heegaard splitting $V \cup_{S} W$ to be

$$
d(V, W)=d\left(\mathcal{D}_{V}, \mathcal{D}_{W}\right) .
$$

Algebraic $8 \mathcal{G}$ Geometric Topology, Volume 7 (2007) 


\subsection{Laminations}

We let $\mathcal{P} \mathcal{M L}(S)$ denote the space of projective measured laminations on $\mathrm{S}$. For a good reference on measured laminations see Bonahon [2], Casson and Bleiler [3], or Hatcher [8].

We will need the following lemma due to Hempel [9], who generalized an argument of Kobayashi [13].

Lemma 2.1 Suppose that $X, Y \subset \mathcal{C}_{S}$, and let $\bar{X}$ and $\bar{Y}$ denote their closures in $\mathcal{P} \mathcal{M L}(S)$. Let $\Phi$ be a pseudo-Anosov map with stable and unstable laminations $\lambda^{ \pm}$. Assume that $\lambda^{-} \notin \bar{Y}$ and $\lambda^{+} \notin \bar{X}$. Then $d\left(X, \Phi^{n}(Y)\right) \rightarrow \infty$ as $n \rightarrow \infty$.

This statement is virtually identical to that of Abrams-Schleimer [1, Theorem 2.4], the only difference being that $X=Y$ there. The same proof carries through.

\subsection{Pants, waves and seams}

Given a surface $S$ and an essential subsurface $Y \subset S$, a wave in $Y$ is a properly embedded arc in $Y$ not homotopic rel $\partial Y$ into $\partial Y$, and with both ends incident to the same boundary component of $Y$ from the same side.

A pair of pants is a three-holed sphere. A pants decomposition $\mathcal{P}=\left\{\alpha_{i}\right\}$ of a surface $S$ is a maximal collection of essential simple closed curves which are disjoint and not parallel; hence (we are assuming genus at least two) $\overline{S-\mathcal{P}}$ is a union of pairs of pants.

A seam in a pair of pants is an essential properly embedded arc connecting distinct boundary components. Up to isotopy rel boundary, a pair of pants has three distinct waves and three distinct seams.

We say an essential curve or a lamination $\alpha \subset S$ traverses or has a wave (seam) with respect to $Y$ if $\alpha$ intersects $Y$ minimally in its isotopy class and a component of $\alpha \cap Y$ is a wave (seam).

A standard outermost bigon argument proves the existence of waves among meridians in a compression body:

Lemma 2.2 Let $V$ be a compression body and let $\mathcal{M}$ be a maximal collection of non-parallel, disjoint meridians on $S=\partial_{+} V$. If $\alpha$ is a meridian of $V$ then $\alpha$ is either parallel to a component of $\mathcal{M}$ or $\alpha$ has a wave with respect to one of the pants components of $S \backslash \mathcal{M}$. 
Let $V, S$ and $\mathcal{M}$ be as above, and let $\overline{\mathcal{D}_{V}}$ denote closure in $\mathcal{P} \mathcal{M L}(S)$. Here and in what follows, by a "seam of a curve system" we mean a seam of a pants component of its complement. We then have:

Lemma 2.3 No lamination $\lambda \in \overline{\mathcal{D}_{V}}$ traverses all seams of $\mathcal{M}$.

Proof Suppose that $\left\{\beta_{i}\right\} \in \mathcal{D}_{V}$ is a sequence of meridians converging to $\lambda \in \mathcal{P} \mathcal{M L}(S)$. If $\lambda \in \mathcal{M}$ then clearly $\lambda$ does not traverse all seams of $\mathcal{M}$. By passing to a subsequence, we may assume that none of the $\beta_{i}$ lie in $\mathcal{M}$. It follows from Lemma 2.2 that all $\beta_{i}$ have a wave in some pants of $S \backslash \mathcal{M}$. Passing to subsequences again we may assume that all of the $\beta_{i}$ have the same wave $w$ in the same pair of pants $Y$.

If $\lambda$ traversed all seams of $\mathcal{M}$, then for large enough index $i$ the curve $\beta_{i}$ would also traverse all these seams. Since one of the seams of $Y$ intersects $w$ essentially it would follow that $\beta_{i}$ self-intersects, a contradiction.

\section{$2.4(g, b)$-Decompositions}

Suppose $\mathcal{A} \subset V$ is a disjoint collection of properly embedded arcs in a compression body $V$ where $\partial \mathcal{A} \subset \partial_{+} V$. We say $\mathcal{A}$ is unknotted if $\mathcal{A}$ can be properly isotoped, rel boundary, into $\partial V$.

The following well-known generalization of bridge position is due to Doll [4]: Suppose that $M=V \cup_{S} W$ and $K$ is a knot in $M$. The knot $K$ is in bridge position with respect to $S$ if $K$ is transverse to $S$ and either

(i) $K \cap S \neq \varnothing$, and both $K \cap V$ and $K \cap W$ are unknotted, or

(ii) $K \cap S=\varnothing, K \subset V$ (without loss of generality), and $V \backslash n(K)$ is a compression body.

If $g=g(S)$ and $b=|K \cap S| / 2$ then we say that $K$ admits a $(g, b)$-decomposition.

Remark 2.4 Our definition of a $(g, 0)$-decomposition is non-standard, due to the additional requirement on $V \backslash n(K)$. In particular this definition makes a $(g, 0)-$ decomposition equivalent to a Heegaard splitting of $E(K)$ of genus $g$.

\subsection{Tunnel number}

Given a knot $K$ in a 3-manifold $M$, a tunnel system for $K$ is a collection $\mathcal{T}=$ $\left\{a_{1}, \ldots, a_{n}\right\}$ of disjoint arcs, properly embedded in $M \backslash K$, such that $M \backslash n(\mathcal{T} \cup K)$ 
is a handlebody. The tunnel number $t(K)$ of the knot is the minimal cardinality $n$ of such a tunnel system.

We note that a knot with tunnel number $t$ has a $(t+1,0)$-decomposition, and that $t+1$ is the minimal genus for which this is possible. Hence it becomes interesting to reduce the genus by one, and ask what $(t, b)$-decompositions such a knot admits.

Of course, if $K$ has a $(g, b)$-decomposition then $K$ also has $(g, b+1)$ and $(g+1, b)-$ decompositions. With a bit more care one can check that any knot with a $(g, b)-$ decomposition also has a $(g+1, b-1)$-decomposition. On the other hand, when going from a $(g, b)$ to a $\left(g-1, b^{\prime}\right)$-decomposition, $b^{\prime}$ may need to grow arbitarily. This follows from Theorem 4.2 applied in the case $(g, b)=(t+1,0)$.

\section{High distance}

We now restate and prove our main theorem on high distance Heegaard splittings for knots.

Theorem 3.1 For any pair of integers $g>1$ and $n>0$ there is knot $K \subset S^{3}$ and a genus $g$ splitting $S \subset E(K)$ having distance greater than $n$.

Consider $S^{3}=V \cup_{S} W$ with the standard genus $g$ Heegaard splitting. Let $\mathcal{C}_{S}, \mathcal{D}_{V}$, and $\mathcal{D}_{W}$ be the corresponding curve and disk complexes. Let $D \subset V$ be a disk cutting $V$ into a solid torus $X$ and a handlebody $Y$ with genus $g-1$. Take $K_{0}$ to be the core of $X$. Thus $V_{0}=V-n\left(K_{0}\right)$ is a compression body and $V_{0} \cup W$ equals $E\left(K_{0}\right)$.

We must find a sequence of compression bodies $V_{n} \subset V$ each homeomorphic to $V_{0}$ so that $\partial_{+} V_{n}=\partial V$ and

$$
d\left(\mathcal{D}_{V_{n}}, \mathcal{D}_{W}\right) \rightarrow \infty
$$

as $n \rightarrow \infty$. The knots $K_{n} \subset S^{3}$ defined by the compression bodies $V_{n}$ will satisfy the conclusion of the theorem.

When $g=2$, which is the case treated by Johnson [10] and Johnson-Thompson [11], $V_{0}$ has a unique non-separating disk and all other disks may be isotoped to be disjoint from it (see, for example, [10, Lemma 11]). Hence $\operatorname{diam}\left(\mathcal{D}_{V_{0}}\right)=2$ and it suffices to find a sequence $V_{n}$ whose non-separating disks get arbitrarily far from $\mathcal{D}_{W}$. In the general case $\operatorname{diam}\left(\mathcal{D}_{V_{0}}\right)=\infty$ so clearly a different construction is needed.

To begin, set $\delta=\partial D$. As shown in Figure 1, extend $\delta$ to a pants decomposition $\mathcal{P}$ whose curves are all meridians of $V$. Likewise, choose a pants decomposition $\mathcal{Q}$ whose boundary curves are meridians of $W$. For later reference we denote by $Q_{1}, \ldots, Q_{g}$ 
the curves of $\mathcal{Q}$ (listed in increasing size) that cross $X$ in the front of the picture. The parallel curves on the back of the picture are denoted by $Q_{2}^{\prime}, \ldots, Q_{g-1}^{\prime}$; note that $Q_{1}$ and $Q_{g}$ are their own parallels. Let $Q_{g+1}$ denote the curve going around the second hole in the picture.

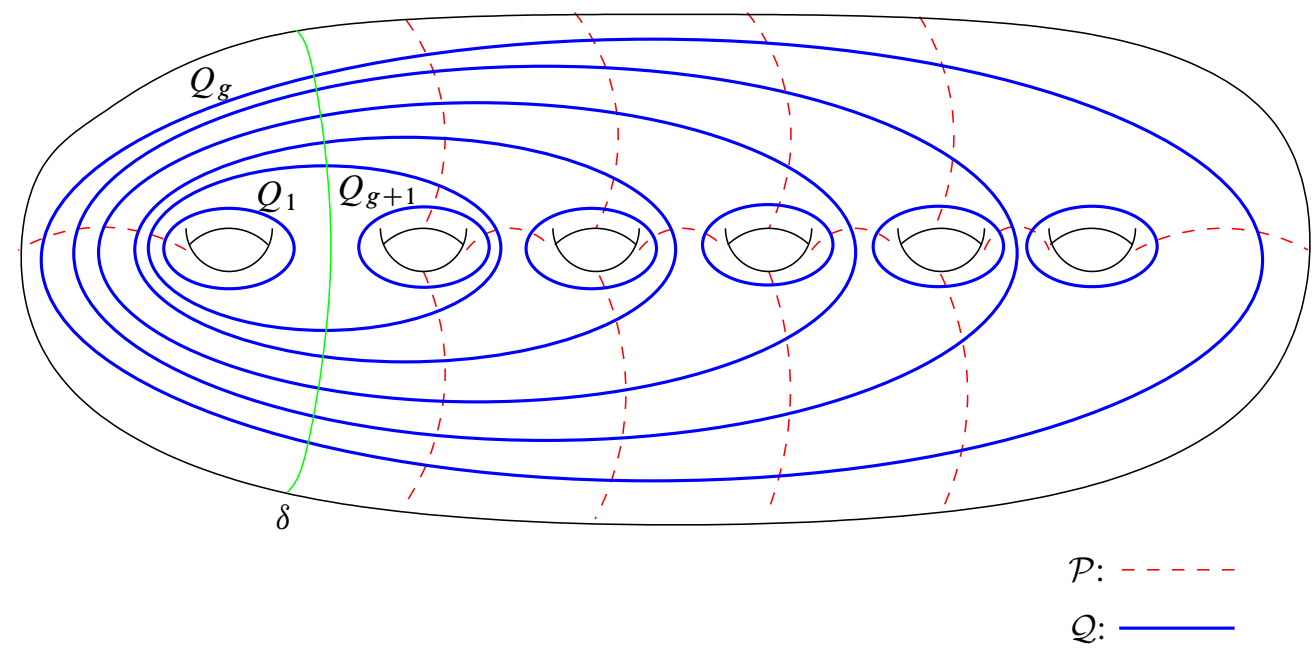

Figure 1: The pants decompositions $\mathcal{P}$ of $V$ and $\mathcal{Q}$ of $W$. Each component $Q_{i}$ of $\mathcal{Q}$ (except the littlest and the biggest) has a symmetric "partner" $Q_{i}^{\prime}$ on the underside of $V$.

Set $S_{0}=\partial Y \cap S$. This is the once-punctured genus $g-1$ surface to the right of $\delta$. Let $\mathcal{P}_{0}=\mathcal{P} \cap S_{0}$ be the pair of pants decomposition of $S_{0}$ induced by $\mathcal{P}$ (note $\mathcal{P}_{0}$ includes $\delta$ ). The important feature of our chosen decompositions is that the curves of $\mathcal{P}_{0}$ traverse every seam of $\mathcal{Q}$ which can be isotoped rel $\mathcal{Q}$ into $S_{0}$.

The construction of $V_{n}$ can be broken up as follows:

Step 1 Find a meridian curve $a \in \mathcal{D}_{V}$ such that:

(a1) $a$ traverses all seams of $\mathcal{Q}$.

(a2) $a$ traverses all seams of $\mathcal{P}_{0}$.

Step 2 Use the curve $a$ to construct a pseudo-Anosov map $\Phi: S \rightarrow S$ which extends over $V$ and whose stable lamination $\lambda^{+}$and unstable lamination $\lambda^{-}$satisfy:

(ג1) $\lambda^{+}$traverses all seams of $\mathcal{Q}$.

( $\lambda 2) \lambda^{-}$traverses all seams of $\mathcal{P}_{0}$. 
Step 3 Show that $d\left(\mathcal{D}_{W}, \Phi^{n}\left(\mathcal{D}_{V_{0}}\right)\right) \rightarrow \infty$ as $n \rightarrow \infty$.

Conclusion Since $\Phi$ extends over $V$, we may define $V_{n}=\Phi^{n}\left(V_{0}\right)$ and $K_{n}=$ $\Phi^{n}\left(K_{0}\right) \subset S^{3}$, so that $\left(V_{n}, W\right)$ is a Heegaard splitting for the knot exterior $E\left(K_{n}\right)$. As desired in the conclusion of the theorem we have:

$$
d\left(\mathcal{D}_{V_{n}}, \mathcal{D}_{W}\right) \rightarrow \infty
$$

\subsection{Proof of Theorem 3.1}

We now carry out the steps outlined above.

Step 1 We will first find a useful curve $\gamma \subset S_{0}$. We will then use two copies of $\gamma$ and a band sum construction to build the desired meridian $a$. So consider a train track $\tau$ adapted to $\mathcal{P}_{0}$ and depicted in Figure 2.

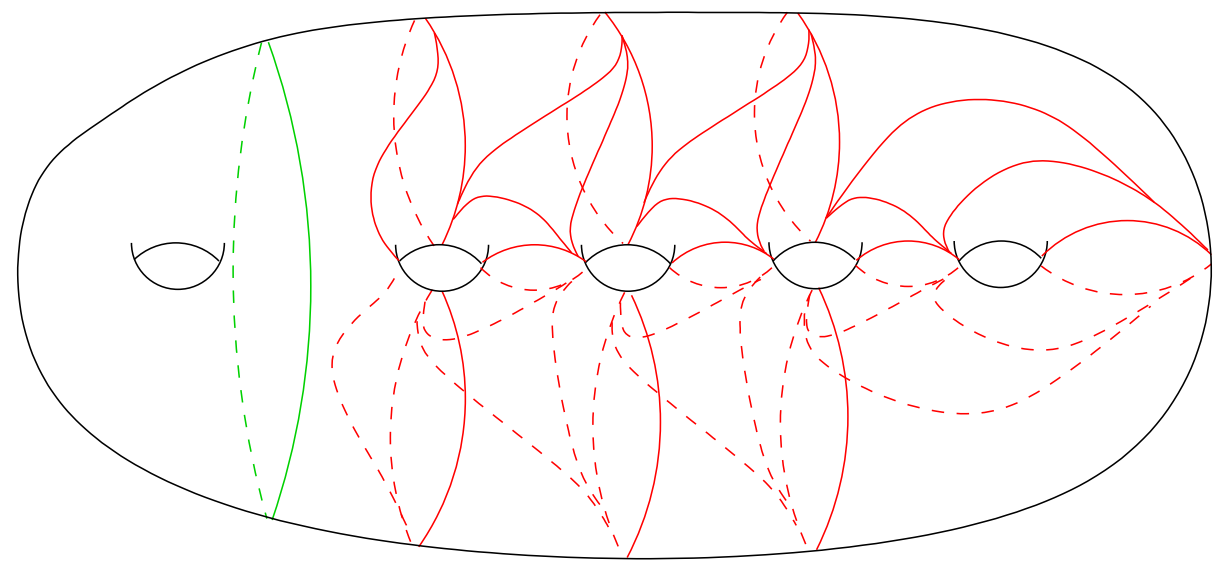

Figure 2: The train track in $S_{0}$

The train track has the following properties:

(1) $\tau$ contains $\mathcal{P}_{0} \backslash\{\delta\}$ and additional branches. These branches traverse all seams of $\mathcal{P}_{0}$, with the exception of the two seams incident to $\delta$.

(2) The entire picture is invariant under a $180^{\circ}$ rotation about the horizontal line meeting $V$ in $g+1$ arcs.

(3) The directions in which incident branches attach to each component of $\mathcal{P}_{0} \backslash\{\delta\}$ on the two sides are consistent, so that there is a train route spiraling around any component of $\mathcal{P}_{0} \backslash\{\delta\}$. 
Note that these conditions determine the track up to the choice of spiraling direction.

Observe that $\tau$ is a "maximal standard train-track" in $S_{0}$, in the sense of PennerHarer [19]. Hence [19, Theorem 3.1.2] tells us that positive measures on $\tau$, modulo scaling, parameterize an open set in $\mathcal{P} \mathcal{M L}\left(S_{0}\right)$. This open set contains a filling minimal lamination (for example, the stable lamination of a pseudo-Anosov homeomorphism) since these are dense in $\mathcal{P} \mathcal{M L}\left(S_{0}\right)$. Since simple closed curves are also dense in $\mathcal{P} \mathcal{M L}\left(S_{0}\right)$ we may approximate this minimal lamination as well as we like by simple closed curves carried on $\tau$. Hence there exists a simple closed curve $\gamma$ carried on $\tau$, traversing every branch at least twice. Note that $\gamma$ has the following properties:

(1) It traverses every seam of $\mathcal{P}_{0}$, again excluding the two seams incident to $\delta$.

(2) It traverses every seam of $\mathcal{Q}$ which is isotopic rel $\mathcal{Q}$ into $S_{0}$.

These properties follow from the corresponding properties of $\tau$, once we observe that the intersections of $\tau$ with $\mathcal{Q}$ are essential, that is, there are no bigons in their complement, and similarly that the intersections of $\gamma$ with $\mathcal{P}_{0} \backslash\{\delta\}$ are essential.

The curve $\gamma$ fails to traverse just four seams of $\mathcal{Q}$, which we denote as follows: Let $\sigma_{1,2}$ be the seam connecting $Q_{1}$ to $Q_{2}$, in the front of $V$. Let $\sigma_{1,2}^{\prime}$ be the seam connecting $Q_{1}$ to $Q_{2}^{\prime}$, in the back of $V$. There are two seams connecting $Q_{1}$ and $Q_{g+1}$ : one in the front, denoted $\sigma_{1, g+1}$, and one in the back denoted $\sigma_{1, g+1}^{\prime}$.

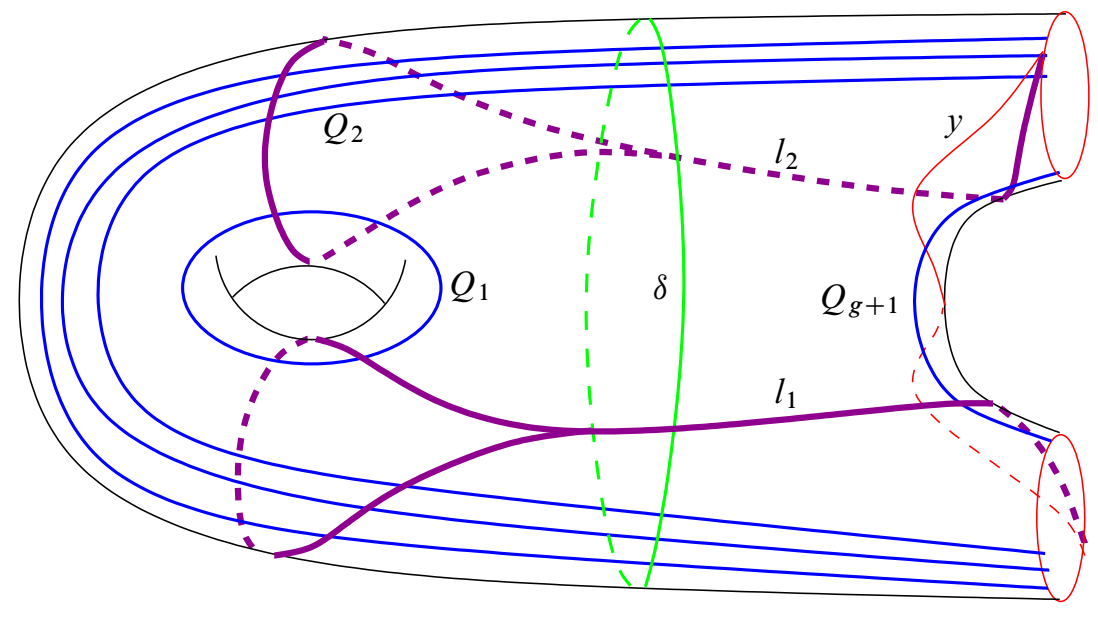

Figure 3: The meridian $a$ is constructed via an enlargement $\tau^{\prime}$ of $\tau$. The figure shows the 2-holed torus $Z$. The added loops are labeled $l_{1}$ and $l_{2}$.

To build the meridian $a$ we add two loops $l_{1}$ and $l_{2}$ to the train track $\tau$ as shown in Figure 3. Let $Z$ denote the 2 -holed torus obtained as the union along $\delta$ of the 1 -holed 
torus in $\partial X$ bounded by $\delta$ and the adjacent pair of pants of $S_{0} \backslash \mathcal{P}_{0}$. Each loop begins inside one of the switches of $\tau$ in $Z$, enters $X$, goes once around the meridian disk, and then returns to the same switch. We define an integer measure on this new train track $\tau^{\prime}$ : Let $\mu$ be the measure on $\tau$ that defines $\gamma$. Hence $2 \mu$ defines two copies of $\gamma$. Now subtract 2 from the weight that $2 \mu$ puts on the branch $y$ of $\tau$ that connects the boundaries of $Z$, and put a weight of 1 on each of the new loops. This measure on $\tau^{\prime}$ defines $a$. One can see that $a$ is a band sum of two copies of the meridian of $X$ along a doubled arc of $\gamma$ - hence it is a meridian of $V$.

Note that the intersection pattern of $\tau^{\prime}$ with the curves $Q_{1}, \ldots, Q_{g+1}$ and $Q_{2}^{\prime}, \ldots, Q_{g}^{\prime}$ has no bigons, and hence all intersections shown are essential (the $Q_{i}^{\prime}$ are not pictured, but lie directly below the corresponding $Q_{i}$ ). One then checks that $\tau^{\prime}$ traverses all four seams that were previously excluded, namely $\sigma_{1,2}, \sigma_{1,2}^{\prime}, \sigma_{1, g+1}$ and $\sigma_{1, g+1}^{\prime}$. Similarly there are no bigons in the intersection pattern with $\mathcal{P}_{0}$, and we see that $\tau^{\prime}$ traverses the two seams of $\mathcal{P}_{0}$ incident to $\delta$. Since $a$ places positive measure on every branch of $\tau^{\prime}$, it traverses all the same seams, and so satisfies conditions (a1) and (a2).

Step 2 Choose two meridians $b, c \in \mathcal{D}_{V}$ so that $b$ and $c$ together fill $S$. (For example: realize $V$ as an $I$-bundle over a compact surface $F$ with exactly one boundary component. Let $e$ and $f$ be $\operatorname{arcs}$ in $F$ so that every nontrivial arc and curve in $F$ meets one of $e$ or $f$ essentially. Then the $I$-bundles over $e$ and $f$ will be the desired disks, because $e \cup f$ cuts $F$ into disks each of which meets $\partial F$ in at most a single interval. See also Kobayashi [12, Proof of Lemma 2.2].) Let $\tau_{a}, \tau_{b}$ and $\tau_{c}$ denote the Dehn twists about $a, b$ and $c$ respectively. Set

$$
\Phi_{0}=\tau_{b} \circ \tau_{c}^{-1}
$$

It follows from Thurston's construction [21] that $\Phi_{0}$ is pseudo-Anosov, with stable/unstable laminations $\lambda_{0}^{ \pm}$. Since $\Phi_{0}$ is a composition of Dehn twists along meridian disks it extends over $V$. Define

$$
\Phi_{N}=\tau_{a}^{N} \circ \Phi_{0} \circ \tau_{a}^{-N} .
$$

Since $a$ is a meridian $\Phi_{N}$ also extends over $V$. The stable and unstable laminations $\lambda_{N}^{ \pm}$of $\Phi_{N}$ are just $\tau_{a}^{N}\left(\lambda_{0}^{ \pm}\right)$. Since $a$ intersects $\lambda_{0}^{ \pm}$(the latter is filling), as $N \rightarrow \infty$ the laminations $\lambda_{N}^{ \pm}$converge to $[a]$ in $\mathcal{P} \mathcal{M L}(S)$. Hence eventually both laminations satisfy conditions (a1) and (a2). Take $\Phi=\Phi_{N}$ for such a large $N$ and take $\lambda^{ \pm}=\lambda_{N}^{ \pm}$. Thus conditions $(\lambda 1)$ and $(\lambda 2)$ are satisfied.

Step 3 Since conditions $(\lambda 1)$ and $(\lambda 2)$ hold, we can conclude via Lemma 2.3 that $\lambda^{-} \notin \overline{\mathcal{D}}_{V_{0}}$ and $\lambda^{+} \notin \overline{\mathcal{D}}_{W}$. The fact that $d\left(\mathcal{D}_{W}, \Phi^{n}\left(\mathcal{D}_{V_{0}}\right)\right) \rightarrow \infty$ now follows from Lemma 2.1. This completes the proof of Theorem 3.1. 


\section{Ruling out simple decompositions}

In order to prove Theorem 4.2 we use a generalization by Tomova [22] of a theorem of hers [23], which is itself a considerable refinement of a theorem of Scharlemann and Tomova [20] (see also Kobayashi and Rieck [14] for another proof).

Theorem 4.1 Let $K \subset S^{3}$ be a knot and $S$ be a Heegaard splitting surface of the exterior of $K$. Suppose that $Q$ is Heegaard splitting surface of $S^{3}$ so that

(1) $K$ is in bridge position with respect to $Q$ and

(2) the genus of $Q$ is less than the genus of $S$.

Then the distance of the Heegaard splitting $S$ is at most $2-\chi(Q-K)$.

Condition (2) is actually stronger than necessary for Tomova's result, but this version suffices for our needs. We are now in position to prove Theorem 4.2:

Theorem 4.2 For any positive integers $t$ and $b$ there is a knot $K \subset S^{3}$ with tunnel number $t$ so that $K$ has no $(t, b)$-decomposition.

Proof Choose a Heegaard splitting $S^{3}=V \cup_{S} W$ of genus $t+1$ and construct the knot $K \subset S^{3}$, as in Theorem 3.1, so that the associated splitting $E(K)=V^{\prime} \cup_{S} W$ has distance $d\left(V^{\prime}, W\right)>2 t+2 b$.

We claim that $K$ has no $(g, c)$-decomposition for any $g \leq t, c \leq b$. For if it did, let $Q$ be the associated splitting surface of $S^{3}$. The genus $g$ of $Q$ is less than that of $S$, so we may apply Theorem 4.1 to obtain $d\left(V^{\prime}, W\right) \leq 2-\chi(Q-K)=2-(2-2 g-2 c) \leq 2 t+2 b$, a contradiction.

Setting $c=0$ we also conclude that $E(K)$ has no splitting of genus less than $t+1$, and hence that $K$ has tunnel number $t$.

\section{References}

[1] A Abrams, S Schleimer, Distances of Heegaard splittings, Geom. Topol. 9 (2005) 95-119 MR2115669

[2] F Bonahon, Geodesic laminations on surfaces, from: "Laminations and foliations in dynamics, geometry and topology (Stony Brook, NY, 1998)", Contemp. Math. 269, Amer. Math. Soc., Providence, RI (2001) 1-37 MR1810534 
[3] A J Casson, S A Bleiler, Automorphisms of surfaces after Nielsen and Thurston, London Mathematical Society Student Texts 9, Cambridge University Press, Cambridge (1988) MR964685

[4] H Doll, A generalized bridge number for links in 3-manifolds, Math. Ann. 294 (1992) 701-717 MR1190452

[5] M Eudave-Muñoz, Incompressible surfaces and (1,2)-knots arXiv: math.GT/0703132

[6] M Eudave-Muñoz, Incompressible surfaces and $(1,1)-k n o t s$, J. Knot Theory Ramifications 15 (2006) 935-948 MR2251034

[7] W J Harvey, Boundary structure of the modular group, from: "Riemann surfaces and related topics: Proceedings of the 1978 Stony Brook Conference (State Univ. New York, Stony Brook, N.Y., 1978)", Ann. of Math. Stud. 97, Princeton Univ. Press, Princeton, N.J. (1981) 245-251 MR624817

[8] A E Hatcher, Measured lamination spaces for surfaces, from the topological viewpoint, Topology Appl. 30 (1988) 63-88 MR964063

[9] J Hempel, 3-manifolds as viewed from the curve complex, Topology 40 (2001) 631-657 MR1838999

[10] J Johnson, Bridge number and the curve complex arXiv:math.GT/0603102

[11] J Johnson, A Thompson, On tunnel number one knots which are not $(1, n)$ arXiv: math.GT/0606226

[12] T Kobayashi, Pseudo-Anosov homeomorphisms which extend to orientation reversing homeomorphisms of $S^{3}$, Osaka J. Math. 24 (1987) 739-743 MR927058

[13] T Kobayashi, Heights of simple loops and pseudo-Anosov homeomorphisms, from: "Braids (Santa Cruz, CA, 1986)", Contemp. Math. 78, Amer. Math. Soc., Providence, RI (1988) 327-338 MR975087

[14] T Kobayashi, Y Rieck, Knot exteriors with additive Heegaard genus and Morimoto's Conjecture arXiv:math/0701765

[15] T Kobayashi, Y Rieck, On the growth rate of tunnel number of knots arXiv: math.GT/0402025

[16] D McCullough, Virtually geometrically finite mapping class groups of 3-manifolds, J. Differential Geom. 33 (1991) 1-65 MR1085134

[17] Y Moriah, H Rubinstein, Heegaard structures of negatively curved 3-manifolds, Comm. Anal. Geom. 5 (1997) 375-412 MR1487722

[18] K Morimoto, M Sakuma, Y Yokota, Examples of tunnel number one knots which have the property “ $1+1=3$ ”, Math. Proc. Cambridge Philos. Soc. 119 (1996) 113-118 MR1356163 
[19] R C Penner, J L Harer, Combinatorics of train tracks, Annals of Mathematics Studies 125, Princeton University Press, Princeton, NJ (1992) MR1144770

[20] M Scharlemann, M Tomova, Alternate Heegaard genus bounds distance, Geom. Topol. 10 (2006) 593-617 MR2224466

[21] W P Thurston, On the geometry and dynamics of diffeomorphisms of surfaces, Bull. Amer. Math. Soc. (N.S.) 19 (1988) 417-431 MR956596

[22] M Tomova, Distance of Heegaard splittings of knot complements arXiv: math/0703474

[23] M Tomova, Multiple bridge surfaces restrict knot distance arXiv: math.GT/0511139

[24] F Waldhausen, Heegaard-Zerlegungen der 3-Sphäre, Topology 7 (1968) 195-203 MR0227992

Department of Mathematics, Yale University

New Haven CT 06520-8283, USA

Department of Mathematics, Technion

Haifa 32000, Israel

Mathematics Institute, University of Warwick

Coventry, CV4 7AL, UK

yair.minsky@yale.edu, ymoriah@tx.technion.ac.il,

S.D.Schleimer@warwick.ac.uk

Received: 25 August 2006

Algebraic 83 Geometric Topology, Volume 7 (2007) 